\title{
La influencia del folclore en los cuentos de la ilustradora Emily Carroll
}

\author{
Folklore's influence on the stories of illustrator \\ Emily Carroll
}

\author{
Emili SAMPER Prunera \\ (Universitat Rovira i Virgili) \\ emili.samper@urv.cat
}

ORCID ID: 0000-0002-9228-237X

\begin{abstract}
The Canadian illustrator Emily Carroll offers in her works a beautiful and, at the same time, terrifying display of female characters and monsters (visible or hidden) that attract the reader both by the form shown and by the background they hide. Many of its stories are related to folklore, whether by the inclusion of traditional elements, the re-elaboration of characters, the appearance of certain monsters or the use of structural elements that we also find in the stories of oral tradition. This article aims to follow this influence of folklore in Carroll's work, taking into account especially the book Through the Woods as well as the webcomics that have a more direct relationship with traditional tales. The aim is to see what elements from tradition can be found in her work and what treatment and use the author makes of them.
\end{abstract}

Keywords: comic, folklore, folktale, PALABras-Clave: cómic, folclore, cuento, webcomic, Emily Carroll
Resumen: La ilustradora canadiense Emily Carroll ofrece en sus trabajos un muestrario bello y, a la vez, aterrador, de personajes femeninos y de monstruos (visibles u ocultos) que atraen al lector tanto por la forma mostrada como por el trasfondo que esconden. Muchas de sus historias tienen relación con el folclore, ya sea con la inclusión de elementos tradicionales, la reelaboración de personajes, la aparición de determinados monstruos o el uso de elementos estructurales que también encontramos en los relatos de tradición oral. Este artículo pretende seguir esta influencia del folclore en la obra de Carroll teniendo en cuenta de manera especial el libro Through the Woods así como los webcómics que guardan una relación más directa con los relatos tradicionales. El objetivo es ver qué elementos procedentes de la tradición se pueden encontrar en su obra y qué tratamiento y uso hace la autora. webcómic, Emily Carroll

«Sure, mama and papa Carroll played some role, but ask what wonder would come from the union of Edgar A. Poe and Edward St. John Gorey and the answer has to be: Emily Carroll»

(Silva, 2014). 


\section{InTROdUCCIÓN: LA OBRA DE EMILY CARROLL}

Ganadora de varios premios (como el Eisner, el Ignatz y el British Fantasy), ${ }^{1}$ Emily Carroll (Londres, Ontario 1983) se dio a conocer en 2010 con sus webcómics, ${ }^{2}$ especialmente con His Face All Red que alcanzó una gran popularidad en la red. ${ }^{3}$ En 2014 publicó Through the Woods (Cruzando el bosque en la edición española de 2015), una antología de cinco historias en las que, con el bosque como elemento común, explora con un grafismo potente la monstruosidad confrontada a los personajes femeninos consiguiendo así impactar (y incomodar al mismo tiempo) al lector. Carroll también ha ilustrado relatos escritos por otras autoras, tales como la revisión del personaje procedente del folclore eslavo hecha por Marika McCoola en Baba Yaga's Assistant (2015) o la adaptación gráfica de la novela Speak (2018) (Cuéntalo en la edición española) de Laurie Halse Anderson.

Una de las características del estilo de Emily Carroll es el uso que hace del formato para sus historias ya que, en su caso, la experiencia de lectura en línea (en los webcómics) no consiste en la publicación en su web de imágenes estáticas sino que ofrece al lector una experiencia más completa, aprovechando la potencialidad del medio:

A final form of fairy-tale graphic narrative adaptations, which also have subversive potential, are webcomics. While many print graphic narratives are available online with the rise of E-reading culture, webcomics are produced and accessible solely online. [...]

Both collections [Andrea L. Peterson and Emily Carroll] alter the reading experience, as the act of turning the page is replaced by clicking the mouse to move to the next screen (Whatman, 2018: 480).

Con la publicación del libro Through de Woods, Carroll dió el salto al papel, pero no con una simple recopilación de historias ya publicadas, hecho bastante habitual en los autores que cambian el formato webcómic por el papel. En este sentido, y dado su éxito previo con los cuentos publicados en internet, la expectación era máxima:

For most creators, a collection of new shorts would be an indulgence - a minor work between larger novels - as such collections rarely sell well. But Carroll's book was highly anticipated, her online presence having built up a devoted fan base who saw the merit of her chosen form (Mazur \& Danner, 2017: 73).

En su caso no se trató, sin embargo, de una simple traslación del formato electrónico (web) al físico (papel), sino que la autora adaptó aquellos relatos que no eran inéditos a la nueva forma (libro) en la que serían leídos. Muestra de ello lo encontramos en el segundo cuento («His Face All Red» [Y la cara toda roja]) ${ }^{4}$ que ya había aparecido con

\footnotetext{
${ }^{1}$ Este artículo se enmarca en una línea de investigación sobre literatura popular que ha recibido financiación del Ministerio de Ciencia, Innovación y Universidades a través del proyecto de I+D PGC 2018-093993-B-100 (MCIU/AEI/FEDER, UE). Asimismo, forma parte del trabajo realizado por el Grup de Recerca Identitats en la Literatura Catalana (GRILC), reconocido y consolidado por la Generalitat de Catalunya (2017 SGR 599).

${ }^{2}$ Un webcómic (término que no aparece en el Diccionario de la lengua española) es una historieta publicada en internet. El uso de este formato facilita la difusión de la obra y, en algunos casos, deriva en la posterior publicación en papel de la misma.

${ }^{3}$ Accesibles en su página web $<$ https://emcarroll.com/> (consultada el 8 de septiembre de 2020).

${ }^{4}$ No se incluye la paginación concreta de los cuentos incluidos en Through the Woods citados en este trabajo ya que las páginas del libro no están numeradas. A pesar de ello, se pueden localizar fácilmente a partir del título. Sí que se incluye la traducción del título de la edición española de 2015.
} 
anterioridad en forma de webcómic. Carroll modificó, en la versión publicada en papel, el número de viñetas que aparecen en una página, con lo cual la versión en webcómic y la versión en papel no presentan la misma experiencia de lectura:

It should be noted that the reprinted stories don't transition from the computer screen to the printed page entirely unscathed. The panel arrangements have to be modified to fit this format, and in a couple cases the storytelling suffers a bit. The main example comes in «His Face All Red», as we see the protagonist descend a hole in the woods. The sequence has more impact when presented as one continuous image. But to be fair, it's doubtful anyone would notice changes like this without having read the digital versions previously (Schedeen, 2015).

Otro aspecto importante en la obra de Carroll es la presencia de protagonistas femeninos en sus cuentos. De manera específica (aunque no exclusiva), en el libro de cuentos sobre el bosque:

Emily Carroll's collection of horror comics, Through the Woods, operates largely on the alienation of the inexplicable experience. More specifically, with one exception, it explores that alienation in women, particularly young women. The struggle for many of these characters is the insidious horror of trauma, and all of the ways that trauma pulls you apart, both from yourself and your community, and leaves you susceptible to further terrors (Horrocks, 2014).

Visualmente, el estilo de Carroll es de una gran potencia y destacan, por encima de todo, la conjunción de tres elementos de diseño: la composición de la página (ya sea impresa o en pantalla), el color y la tipografía utilizada:

Carroll's web comics have long established her prowess for design. Paper and pages, instead of pixels and clicks, make Through the Woods a different beast; however, her instinct for pacing and the structure of the page remains intact. For Carroll, it's composition all the way down. Every page of Through the Woods is laid out like trail of breadcrumbs for the reader's eyes to follow. Carroll's colours and cartooning share equal space with her lettering. Her desiccated letters stand in contrast to the vibrancy of sumptuous reds, midnight blues and her always smothering blacks. Carroll treats each graphic element (pencils, inks, colours and letters) as a component of the page. Each bit fits its composition and, oh by the way, serves the story (Silva, 2014).

El uso del color no es nunca anecdótico sino que tiene una función concreta dentro de las historias:

One of the aspects of the whole that powers Through The Woods is its lettering. Carroll's lettering has a handwritten character of its own, and oftentimes the twisting bending nature of the letters and words drive the composition of the pages as a whole, which allows Carroll to move effortlessly through sometimes complex layered montage pages. [...] Carroll's color palette also demonstrates command of the medium. Oranges and reds bleed out of bright whites, themselves dusted across dark mysterious black shadows. Her color choices function both in spooky service to the atmospheric mood and narration, and also in aid of the stories' larger thematic threads (Horrocks, 2014). 
Un buen ejemplo del uso de ambos elementos (tipografía y color) al servicio de la historia es el cuento «A Lady's Hands are Cold» (La dama de las manos frías), también publicado en Through de Woods, en el cual se reproduce la letra de la canción que canta la esposa difunta asesinada por su marido. Así, la letra llena las viñetas de las páginas consiguiendo crear un ambiente obsesivo que agobia y persigue a la protagonista, con el color azul como marca de la historia en la cual se basa (como veremos), en contraste con la aparición del color rojo.

Emily Carroll ha creado un universo propio con sus historias. Gráficamente hemos apuntado algunos de los rasgos característicos de su obra. Este estilo personal se puede reconocer también si consideramos su obra como un conjunto. Me refiero a la relación que podemos establecer entre historias diferentes. Veamos a continuación las dos muestras más representativas.

La primera pertenece precisamente al libro Through the Woods. Se trata del quinto cuento, titulado «The Nesting Place» (El nido), que cierra el libro y que es una muestra excelente de la presencia de monstruos femeninos en la obra de Carroll. En este cuento aparece el personaje de Rebecca, la prometida del hermano de la protagonista (Bell) que, de joven se perdió en el bosque durante días. Cuando reapareció, su comportamiento había cambiado. Este mismo personaje lo encontramos en el webcómic All Along the Wall también del 2014. ${ }^{5}$ En esta ocasión, Rebecca cuenta una historia de miedo a una niña (Lottie) y le dice que fue ella misma la que se perdió en el bosque. El final inquietante de este cuento (con la imagen de Rebecca peinando a Lottie) se puede ver como un anticipo del desenlace del cuento incluído en Through the Woods. En este sentido, el webcómic se situaría cronológicamente antes que el cuento en papel. Ambas historias comparten personaje y también el trasfondo de la historia, con la presencia amenazadora del monstruo (femenino), más explícito (y violento) en el cuento que en el webcómic. En cualquier caso, la lectura conjunta de ambos relatos enriquece la experiencia lectora.

El segundo ejemplo lo encontramos en When I Arrived at the Castle (2019) (La noche que llegué al castillo en la versión española de 2020), el último libro publicado en papel por Carroll, que nos muestra una historia lésbica de vampirismo. En esta ocasión, el elemento común no es la presencia de ningún personaje concreto sino el uso de un recurso que la autora desarrolla de manera diferente según el medio utilizado. En un momento dado, la protagonista de la historia (una mujer-gato) encuentra una habitación llena de puertas y tiene que escoger cuál quiere abrir. Cada puerta esconde un relato diferente, en este caso, presentado en forma textual por parte de Carroll. Estos relatos, que la protagonista descubrirá, serán el elemento clave para entender el final de la historia y la relación entre las protagonistas. Carroll utiliza el mismo recurso (la elección de una puerta que abre una nueva historia) en el webcómic The Worthington (2018). ${ }^{6}$ En esta ocasión, es el lector el encargado de elegir cuál de las seis puertas quiere abrir, cada una de las cuales está identificada con un número: 8 (un banquete); 65 (una mujer sin cabeza); 504 (una historia en primera persona con la prohibición de girarse); 1.002 (unas miradas en el espejo); 21 (una pareja que se ha divertido y la presencia de un cadáver); 223 (una mujer que dice que no hay nada que ver en esta habitación). Si en el relato vampírico, publicado en papel, la apertura de cada puerta,

\footnotetext{
${ }^{5}$ Disponible en $<$ http://emcarroll.com/comics/wall/> (consultada el 8 de septiembre de 2020).

${ }^{6}$ Disponible en $<\mathrm{http}$ ://www.emcarroll.com/comics/worthington/> (consultada el 8 de septiembre de 2020).
} 
por parte de la protagonista, nos ofrece la lectura de un relato (texto), en esta ocasión es el lector quien se convierte en el protagonista ya que debe elegir qué puerta quiere abrir. Y la técnica utilizada también es diferente: no se trata de un relato escrito (como ocurre en el libro impreso) sino de un desenlace gráfico que aprovecha tanto en la forma como en la manera de acceder (haciendo clic) las posibilidades de creación artística que ofrece internet.

\section{EMILY CARRoll: CÓMIC y FOLCLORE}

El presente trabajo pretende analizar la influencia del folclore en la obra de Emily Carroll teniendo en cuenta los dos formatos en los cuales la autora ha publicado (webcómic y papel) ya que, como se ha visto en la introducción precedente, el formato elegido y su uso no es casual en la autora canadiense. El corpus a tener en cuenta está formado por los webcómics que Carroll ha publicado en su web y por dos obras en papel: Through the Woods (2014) y Baba Yaga's Assistant (2015), esta última realizada conjuntamente con Marika McCoola que se encarga del guion. El objetivo es ver qué elementos procedentes de la tradición se pueden encontrar en esta obra de Carroll y qué tratamiento y uso de los mismos hace la autora.

Antes de empezar este análisis, vale la pena revisar someramente algunos conceptos sobre la relación entre el folclore (los cuentos, sobre todo) y el cómic. En la revisión histórica hecha por Daniel Peretti sobre los cómics como género folclórico encontramos una afirmación ya clásica de Lauretta Bender y Reginald S. Lourie del año 1941, según la cual: "Comic books can probably be best understood if they are looked upon as an expression of the folklore of this age. They may be compared with the mythology, fairy tales, and puppet shows, for example, of past ages» (Bender \& Lourie, 1941 citados por Peretti, 2016: 109-110).

Sin entrar ahora en el detalle de este interesante debate teórico sobre la consideración (o no) del cómic como un género folclórico, sí conviene destacar dos aspectos de esta relación entre folclore y cómic: (1) la potencialidad que ofrecen las viñetas en las adaptaciones de cuentos, ya que: «comics and graphic novels offer multimodal media for the adaptations of fairy-tale narratives, characters, motifs and iconography» (Whatman, 2018: 474); (2) y el hecho de que "horror is a popular genre for graphic narrative adaptations» (Whatman, 2018: 476). Ambos aspectos hay que tenerlos en cuenta cuando se analiza la obra de Emily Carroll ya que ésta se puede leer en pantalla y también, parcialmente, en papel, y el horror y el terror son dos de los elementos característicos de su producción artística. En cuanto a la relación directa con el folclore, la misma artista canadiense reconoce que: «I think when it comes to outright influences in my own comics though, I'm a lot more influenced by children's books and fairy tales, not necessarily just other comics» (Carroll entrevistada por Collins, 2011). Estas influencias populares afloran (en diferentes niveles) en su obra, como veremos a continuación.

Para ver qué elementos procedentes de la tradición se pueden encontrar en la obra de Emily Carroll se ha divido el corpus analizado en dos grupos diferentes. En primer lugar se analizarán las versiones folclóricas, esto es, las que tienen una relación más clara (y explícita) con cuentos tradicionales. En segundo lugar, se tendrán en cuenta aquellas historias que se acercan, en cierto modo, a los relatos de tipo tradicional sin que se puedan adscribir de manera específica a ninguno en concreto para las cuales se aplicará la etiqueta de versiones «folclorescas». 


\subsection{Versiones folclóricas}

Para analizar las versiones más próximas al folclore en la obra analizada de Emily Carroll se tendrán en cuenta las aportaciones teóricas de Aldred (2016) y de Smith (2007) sobre la relación entre la fuente original (el cuento, en este caso) y la versión (en webcómic y en cómic) elaborada por la autora.

Así, Grantham Aldred (2016) propone tres categorías para los cómics relacionados con los cuentos de hadas. Los cómics pueden: recontar (retelling), adaptar (adaptation) o imitar (pastiche) el relato original. ${ }^{7}$ Por otro lado, Kevin Paul Smith (2007: 10) identifica, basándose en las categorías de Genette, ocho usos intertextuales de los cuentos de hadas:

1. Authorised: Explicit reference to a fairytale in the title.

2. Writerly: Implicit reference to a fairytale in title.

3. Incorporation: Explicit reference to a fairytale within the text.

4. Allusion: Implicit reference to a fairytale within the text.

5. Re-vision: putting a new spin on an old tale.

6. Fabulation: crafting an original fairytale.

7. Metafictional: discussion of fairytales.

8. Architextual/Chronotopic: «Fairytale» setting/environment.

Smith denomina a estas categorías «elementos» para reflejar la complejidad de la intertextualidad y evidenciar que pueden aparecer en diferentes combinaciones. Este autor, de hecho, aplica estos elementos en versiones escritas (textuales) de cuentos, pero su categorización es aplicable también a versiones no estrictamente textuales, como las que ofrece Carroll en su producción gráfica en formato de cómic.

Entrando ahora en el corpus a analizar, la primera historia a tener en cuenta es precisamente la primera publicada por Emily Carroll en su web, que data de 2010 y se titula The Hare's Bride. ${ }^{8}$ Se trata de una versión del cuento del mismo título publicado por los hermanos Grimm en los Kinder- und Hausmärchen. Concretamente, el número 66 «Häsichenbraut». Se trata de un buen ejemplo de cómic que «recuenta» el relato original, ya que, según Aldred:

The simplest of these forms is the direct retelling. In the direct retelling, the setting and characters are the same as in the original source material. [...] These direct retellings sometimes exists as individually published comics and sometimes exists as parts of larger collections. An example of the former is David Wenzel and Douglas Wheeler's Fairy Tales of the Brothers Grimm (1995) (Aldred, 2016: 423).

Este tipo de elaboración no altera el contenido de la historia, pero «attempt to faithfully retell folktales in the comic medium» (Aldred, 2016: 423), que es exactamente lo que hace Carroll aquí. Por otro lado, la referencia explícita al cuento de los hermanos

${ }^{7}$ La propuesta de Aldred es aplicable, de hecho, a cualquier otra obra artística (y literaria) modificada en función del soporte utilizado o del público destinatario. En el caso que nos ocupa, resulta especialmente útil para clasificar los cómics de Carroll que tienen una relación directa (y explícita) con la fuente en la cual se basan.

${ }^{8}$ Disponible en $<$ http://emcarroll.com/comics/haresbride.html $>$ (consultada el 8 de septiembre de 2020). 
Grimm que encontramos en el título nos permite aplicar el primer uso intertextual de la propuesta de Smith (authorised), según el cual:

Element one, the fairytale as an authorised intertext is the most obvious use of fairytale as an intertext. The use of a proper name of a fairytale in the title acts as an authorial sanction that the text is to be understood in its relevance to a prior, pre-existing fairytale. [...]

An explicit intertextual reference within the title, then, sets up a whole set of mechanisms whereby the reader automatically assumes that this intertextual reference is somehow relevant to the following text, the default setting may indeed be to assume that the new text is a version of the earlier, identically titled text. This is the most obvious sign of an author explicitly indicating the intertextual relation between his or her text and a predecessor (Smith, 2007: 12-14).

Esta dependencia respecto la fuente original se ve claramente en los diálogos de la historia, ya que reproducen los que aparecen en el cuento recogido por los hermanos Grimm. Este hecho no nos debe sorprender ya que nos encontramos con un relato el desarrollo del cual depende de estos elementos textuales. Me refiero a las fórmulas usadas por la liebre protagonista: «Come here, maiden. Sit on my tail and come away to my little hut» y las tres variantes de «Open the door! Open the door! The wedding guests are merry/waiting/ hungry!». Esta coincidencia también la encontramos en el sonido que la protagonista hace para asustar a la liebre al principio de la historia: «Shoo!» (Grimm, 2007: 321-323).

Dentro de este grupo de versiones folclóricas podemos incluir dos historias (tres, si tenemos en cuenta la conclusión o resumen) del libro publicado en 2014, Through the Woods, con el que la autora dio el salto de la pantalla al papel y logró un mayor impacto y reconocimiento.

El primer cuento incluido en el libro (después del preámbulo) es «Our Neighbor’s House» (La casa del vecino) que cuenta la historia de tres hermanas (Mary, Beth y Hannah) que se quedan solas en casa cuando su padre se marcha de caza. El padre les dice que si no ha vuelto en tres días, se vayan a la casa del vecino. La hermana mayor, primero (Mary), y la pequeña, después (Hannah), desaparecen tras haber visto un hombre con sombrero y una sonrisa que deja al descubierto todos sus dientes. Beth, la hermana mediana, se queda sola y decide finalmente obedecer a su padre y marcharse.

Cada hermana tiene una capa de abrigo de un color diferente y este es precisamente el elemento del cuento a destacar: Mary (verde guisante), Hannah (marrón) y Beth (rojo). Las capas desaparecen cuando lo hacen sus propietarias (desaparición que no vemos como lectores). En el último caso sí somos testigos de cómo Beth se pone la capa roja y sale de casa. El color de la capa y esta acción nos remiten de manera evidente al personaje de Caperucita Roja (Little Red Riding Hood), que corresponde al tipo 333 del catálogo internacional ATU (Uther, 2004). La representación gráfica que hace Carroll no ofrece dudas, en este sentido.

Esta referencia a Caperucita no se trataría, estrictamente, de una nueva manera de explicar el cuento, sino que correspondería a la segunda categoría propuesta por Aldred, la adaptación (adaptation), según la cual:

Adaptations take folktales or fairy beliefs as inspiration but adapt the setting or the characters in some way. These comics often blend genre conventions of folktales and 
fairy tales with the conventions of other genres either in form or in style. An example of the former comes in Neil Gaiman and Charles Vess's work Stardust (1997). [...] Another style of adaptation comes in the expansion of established material (Aldred, 2016: 423). ${ }^{9}$

Un paso más, en este sentido, es el que propone Dokou (2017) en su análisis del cuento de Carroll, ya que identifica al misterioso personaje con sombrero (y al lobo de la conclusión) con una conocida figura del folclore norteamericano, como es la del wendigo. ${ }^{10}$

El personaje de Caperucita Roja aparece de nuevo en la conclusión (o resumen) del libro («In Conclusion» [En resumen]). Como señala Silva (2014): «It's in the introduction and the conclusion when Carroll is at her mosto wolfish; in the Latter her love for Fairytales, especially Little Red Riding Hood, is almost too Obvious to note». En la conclusión se reproduce el viaje de Caperucita desde la casa de su padre a la de su madre (y no de la abuela, en este caso) y la advertencia del peligro que supone la presencia de lobos en el bosque y en un contexto nocturno. Caperucita llega a casa y se acuesta, contenta de haber llegado a su destino sin haberse encontrado con el lobo. Es entonces cuando una sombra, desde la ventana, ${ }^{11}$ le recuerda que deberá cruzar el bosque más a menudo. Para no cruzarse con el lobo, deberá tener suerte cada vez que lo haga, mientras que el lobo sólo necesitará tener suerte en una única ocasión. Este final enlaza, visualmente, con el preámbulo del libro, cuando vemos a una niña leyendo en su cama antes de acostarse:

If Carroll lets the reader dangle at the edge of the bed in the introduction, her conclusion shows what happens when borders get crossed and characters choose to venture through the woods. Spoiler: nothing happens. Carroll plays with the reader's experience having read Through the Woods and manages to pull the wool over the wolf, if only for a moment (Silva, 2014).

La presencia de Caperucita en Through the Woods tiene, de esta manera, un tratamiento doble en el libro ya que, a diferencia de «Our Neighbor's House» (que es una adaptación, como se ha visto), en la conclusión nos encontramos con un caso de retelling (siguiendo a Aldred, 2016) y también de revisión: «putting a new spin on an old tale» (siguiendo a Smith, 2007), ya que, a pesar de seguir la estructura básica del relato tradicional, Carroll añade un tratamiento final más terrorífico que consigue dejar al lector con la incertidumbre ante el futuro del personaje.

La segunda historia que podemos incluir en este grupo de versiones folclóricas es también la segunda que encontramos en el libro Through the Woods. Se trata de «A

\footnotetext{
${ }^{9}$ En la tercera categoría (pastiche), que no se aplica a ninguna historia de Carroll, personajes de diferentes cuentos interactúan entre ellos e, incluso, con personajes de otros géneros folclóricos. Aldred pone como ejemplos de esta categoría la serie The Sandman (1988-1996) de Neil Gaiman y, sobre todo, la serie Fables (2002-2015) de Bill Willingham, donde vemos desfilar e interactuar toda una serie de personajes procedentes del folclore de diferentes culturas.

${ }^{10}$ El wendigo es una criatura mitológica propia de las tribus Algonquin de las Primeras Naciones ubicada, entre otros lugares, en la costa este de Canadá (recordemos que Carroll es canadiense). Se representa habitualmente como un espíritu que ha poseído a un ser humano y que lo induce a cometer asesinatos, canibalismo y otros comportamientos similares.

${ }^{11}$ En un primer momento, el lector identifica la presencia de la luna en esa ventana, pero, en la viñeta siguiente, se revela que se trata de uno de los ojos del lobo.
} 
Lady's Hands are Cold» (La dama de las manos frías) que podemos identificar como una adaptación (adaptation) del cuento Maiden-Killer (Bluebeard), correspondiente al tipo cuentístico ATU 312. En la versión de Carroll, y a diferencia del relato tradicional, el papel de asesino del marido lo adopta el fantasma de la mujer asesinada y no aparece la prohibición de no poder abrir una habitación cerrada. Sí que aparecen, en cambio, otros elementos que nos permiten relacionar el cuento de Carroll con Bluebeard, como son el inicio de la historia (la promesa entre la protagonista y el hombre rico propietario de la mansión) y la presencia del color azul (caracterizando al marido, así como a las paredes de la casa donde se encuentra el cadáver troceado de la anterior mujer y este mismo cadáver en forma de fantasma). Nos encontraríamos, así, en el terreno de la alusión (allusion), que no es otra cosa que una referencia implícita al cuento, desde un punto de vista intertextual, en la que los personajes son identificados por sus acciones (Smith, 2007: 19, 23).

Vale la pena, asimismo, destacar dos elementos propios del cuento de Carroll que acentúan el carácter marcadamente terrorífico del relato. En primer lugar, el hecho de que el cadáver de la mujer anterior del marido rico esté troceado y sea la protagonista la encargada de reconstruirlo, en una especie de operación similar a la realizada por el doctor Frankenstein y su conocida criatura. ${ }^{12}$ En segundo lugar, el leitmotiv que lleva la protagonista a descubrir el cadáver escondido entre las paredes de la casa es la canción que este canta. ${ }^{13}$ Se trata de un elemento que no se encuentra reflejado en el tipo 312, pero que sí aparece en otros cuentos en los que es precisamente la canción cantada por un personaje asesinado lo que permite recuperar el cadáver y descubrir la identidad del culpable. Es el caso, por ejemplo, de la canción que encontramos en el tipo 720 The Juniper Tree («Mi madre me ha matado, mi padre me ha comido» en las versiones españolas) que permite que se haga justicia y que, en este caso, la madrastra reciba un castigo por haber asesinado a su hijastro y haberlo dado a su marido para que se lo coma. En el cuento de Carroll, la canción tiene una función totalmente opuesta ya que deriva en la reconstrucción del cadáver y la posterior persecución de la protagonista. Hecho que enlaza con un final que, tal y como remarca Schede (2015):

The ending to this story is especially striking. It sets the tone for the rest of the book as it establishes that there are no happy endings for the protagonists. Only fear or loss or death or some combination of the three await. Carroll also shows a tendency to end her tales abruptly. Not to the extent of something like Optic Nerve, but in a way that enhances the tension and dread.

Dentro de estas versiones folclóricas podemos incluir un ejemplo que combina el uso autorizado (authorised), con la referencia explícita al título, con la adaptación (adaptation). Se trata del libro Baba Yaga's Assistant (2015). En esta ocasión no es una obra original de Carroll sino un trabajo realizado con Marika McCoola que es quién desarrolla la historia mientras que Carroll se ocupa de la parte gráfica. ${ }^{14}$ Este no es un detalle menor, ya que en ambos casos se ve (como es lógico) la mano de Carroll y se

\footnotetext{
${ }^{12}$ En este caso, sin embargo, con un componente maravilloso y no científico, ya que la unión de las partes del cuerpo se hace con una cinta roja y no con una operación quirúrgica.

${ }^{13}$ Y que Carroll resuelve, gráficamente, de manera magistral con el uso del color y de la tipografía, como se ha explicado en la introducción a modo de ejemplo del estilo de la autora canadiense.

${ }^{14}$ Es el mismo tipo de colaboración (o trabajo conjunto) que encontramos también en la adaptación gráfica de la novela Speak (2018) de Laurie Halse Anderson.
} 
plasma su característico estilo gráfico, pero pasado por el tamiz de la historia (y el guion) de otra autora. Esto no desvirtúa su papel como creadora, pero sí evidencia la dependencia respecto la parte textual de la obra. Tampoco hay que olvidar, en este sentido, el público destinatario de la historia (un público infantil/juvenil), lo que también modula la forma en que Carroll la ilustra. Al contrario de lo que encontramos en las historias elaboradas íntegramente por ella y dirigidas a un público adulto, en este caso la vertiente que potencia la visualización del terror y el horror queda muy matizada.

En cualquier caso, Baba Yaga's Assistant nos presenta explícitamente este personaje proveniente del folclore eslavo en el título de la obra y nos muestra un abanico de sus características definitorias a lo largo de la historia. Lo que interesa destacar es como Carroll inserta gráficamente en el libro diferentes escenas, a modo de flashbacks, que recuperan el pasado de la protagonista (Masha) y que enlazan con elementos cuentísticos del folclore ruso, de manera que «Rhythmic omniscient narration and ornate panel borders for the flashback scenes spotlight the story's Russian folkloric roots» (Gall, 2015: 110). Estos cuentos aparecen en los siete primeros capítulos de la obra:

I. «Baba Yaga's Assistant». Aparece Masha con su abuela que le cuenta un cuento protagonizado por Baba Yaga y una muñeca rusa y caracterizado gráficamente con elementos visuales tradicionales rusos.

II. «Into the Woods». Masha recuerda historias sobre la Baba Yaga que le serán de utilidad para superar la prueba inicial a la que es sometida.

III. «The First Test». Con la aparición de una muñeca rusa (Matryoshka), se nos presenta un recuerdo de la protagonista con su abuela. Después, serán precisamente las muñecas rusas las que ayudarán a la protagonista con la limpieza de la casa de Baba Yaga.

IV. «Complications». En el cuento que la protagonista recuerda de su infancia aparece la escena del beso que sirve para desencantar al príncipe. En el presente, Misha besa un hueso que se transforma al instante en un talismán.

V. «A Practical Examination». Nos presenta a la protagonista con su abuela y su padre y que recrea un accidente sucedido en el jardín. La abuela le dice que las cosas rotas se pueden arreglar y curar ya que nada es tan difícil o está tan sucio como para no poder ser arreglado o limpiado.

VI. «The Second Test». En el cuento se utiliza un escurridor para bañar a unos animales. Misha repite la operación y tapa con barro la parte inferior de la pica para que no se escurra el agua.

VII. «The Third Test». Misha y su abuela recuerdan el entierro de su madre donde la abuela le daba galletas de jengibre.

La función de estos relatos que aparecen en distintos momentos clave de la historia es la de hacer de guía de la protagonista: los recuerdos de su abuela (una figura muy importante en su vida, por la muerte prematura de su madre y porque es ella quien le ofrece el conocimiento del mundo folclórico de Baba Yaga) servirán de pistas para superar las diferentes pruebas que se encuentra la protagonista, precisamente en su camino por servir a Baba Yaga.

\subsection{Versiones «folclorescas»}

En la obra de Carroll encontramos historias cercanas (por temática, personajes, estructura o elementos) al folclore. Pero, al contrario de lo que hemos visto en los ejemplos 
precedentes de las versiones folclóricas, estos cuentos no se pueden adscribir a ningún relato folclórico concreto. Es por ello que para este segundo grupo de historias de la autora canadiense se aplicará el concepto folkloresque de Michael Dylan Foster: ${ }^{15}$

Simply put, the folkloresque is popular culture's own (emic) perception and performance of folklore. That is, it refers to creative, often commercial products or texts (e.g., films, graphic novels, video games) that give the impression to the consumer (viewer, reader, listener, player) that they derive directly from existing folklore traditions. In fact, however, a folkloresque product is rarely based on any single vernacular item or tradition; usually it has been consciously cobbled together from a range of folkloric elements, often mixed with newly created elements, to appear as if it emerged organically from a specific source. In some cases, the form rather than the contents provides this veneer of folklore; the folkloresque can reference folklore in either langue or parole or both (Foster, 2016: 5).

Foster (2016: 5-9) añade que un aspecto importante de este tipo de elementos es que están imbuidos de un sentido de autenticidad, tal y como los percibe el consumidor y/o el creador, y que deriva precisamente de su asociación con el folclore auténtico (o real). En este sentido, y es lo que nos interesa en el caso de Carroll, hay que tener en cuenta que si el producto en cuestión se remonta (o se asocia) a una tradición oral que resulta genuina es menos importante que el hecho que los consumidores sientan (o aprecien) que se trata de un producto folclórico.

Hay tres categorías aplicables al concepto de folkloresque: la integración (integration), la representación (portrayal) ${ }^{16}$ y la parodia (parody) ${ }^{17}$ que se pueden superponer y cruzar y que no se excluyen entre ellas. La que podemos aplicar a esta parte de la obra de Carroll es la primera:

This line of inquiry considers how popular culture products integrate or stitch together folkloric motifs and forms to make a product that appears to be inspired directly by one or more specific traditions. The folkloresque of this mode works through the mechanisms of allusion and pastiche, a hodgepodge suturing of bits and pieces of other things to create a coherent new whole. [...]

Within this form of the folkloresque, older folkloric and newly created elements are exposed to mutual contamination; the folkloresque may not be folklore but it is also not completely invented.

The use of folklore in this way, in terms of both form and content, can be unconscious (part of the folklore-creative process itself) or it can be very much an intentional act (Foster, 2016: 15).

${ }^{15}$ En un sentido muy parecido al del concepto folkloresque de Foster, podemos situar el elemento número ocho de la propuesta intertextual de Smith (2007: 48): Architextual/Chronotopic.

${ }^{16}$ Se trata de la «imagen» que sobre el folclore y los folcloristas (los recolectores y estudiosos de la materia) se tiene en la sociedad y que se refleja en la cultura popular. Foster pone como ejemplo de este tipo de productos películas como Candyman (1992), videojuegos como Folklore o series de televisión como MythBusters, Supernatural o The Folklorist que presentan una imagen de los folcloristas que no se corresponde con la real (Foster, $2016: 16-17)$.

${ }^{17}$ Una parodia, en este sentido, no debe ser necesariamente humorística, pero sí tiene que ser consciente de que imita y de que hace referencia a algún elemento procedente del folclore. Detrás hay una apropiación intencionada de motivos y estructuras folclóricas que pueden ser utilizadas con intención crítica. Ejemplos de este tipo de producto son las películas Enchanted (2017), Princess Bride (1987) y la franquicia Shrek (Foster, 2016: 18-19). 
De manera concreta, podemos aplicar el concepto de folkloresque a cuatro cuentos de Carroll (tres webcómics y un cuento incluído en Through the Woods):

Anu-Anulan \& Yir's Daughter (webcómic del año 2011) ${ }^{18}$ Cuenta la historia de amor entre la diosa Anu-Anulan y Yorenn, hija de Yir. El cuento toma el aspecto de un relato mitológico: vemos la sucesivas transformaciones de la diosa adoptando las formas de cuervo, niña y guerrero para pedir a Yorenn un trozo de su cabello plateado. Aunque podemos relacionar el nombre de la diosa (Anu-Anulan) con el nombre de la diosa celta y del dios del cielo de la mitología sumeria (Anu), en este caso no se trata de ninguna adaptación.

The Prince \& The Sea (webcómic del año 2011). ${ }^{19}$ Un príncipe conoce a una doncella que vive en un lago. Los dos se explican cómo son sus reinos, pero no pueden compartir sus mundos antagónicos porque él necesita aire y ella agua para poder vivir. El príncipe idea un plan para construir un foso y reunir los dos reinos, pero es asesinado por su tío que quiere acceder al trono. La doncella encuentra el cadáver del príncipe y se lo lleva a su reino acuático. El cuento recuerda claramente las historias entre humanos y seres acuáticos femeninos que suelen compartir un final trágico con la ruptura de la relación entre ambos, habitualmente por haber roto una promesa. ${ }^{20}$ Gráficamente, resulta muy interesante la solución adoptada por la autora en el desenlace de la historia ya que se trata de un buen ejemplo del potencial del formato webcómic uniendo contenido y forma: la última página nos presenta el descenso del príncipe (ya difunto) al fondo del agua, arrastrado por su amada. Y el lector efectúa este descenso de manera literal haciendo bajar la pantalla de su ordenador (o dispositivo móvil) hasta el final, para llegar al fondo del agua (y de la historia). ${ }^{21}$

The Three Snake Leaves (webcómic del año 2013). ${ }^{22}$ Se trata de una historia de amor en la que aparece un elemento mágico: tres hojas que tienen el poder de resucitar. Dos enamorados se prometen mutuamente acompañarse a la cripta cuando uno de ellos muera. Muere primero ella con lo cual él es enterrado vivo. Aparece una serpiente, él la hiere y otra serpiente le cura las heridas con tres hojas. Cuando estas caen sobre el rostro de ella, vuelve a la vida. Los dos enamorados piden auxilio y el cuento nos ofrece dos opciones: la salida de ella o la de él. Ambas desembocan en el mismo desenlace aunque con matices diferentes. Es el lector quién debe decidir qué salida elige. Se trata, de nuevo, de una muestra de las características que ofrece el formato webcómic ya que, según la decisión que tome el lector, se abrirá una u otra página web con distinto contenido.

«His Face All Red» (Y la cara toda roja) (incluído en Through the Woods y publicado con anterioridad como webcómic en el año 2010). ${ }^{23}$ De hecho, como ha

\footnotetext{
${ }^{18}$ Actualmente ya no se encuentra disponible en la web de la autora. Sobre esta cuestión, Wejebe (2019) apunta que una posible explicación de su desaparición puede ser que la historia no sigue el tono de horror de los cómics de la autora, ya que se trata de una «charming love story with fairy tale overtones».

${ }^{19}$ Disponible en $<$ http://emcarroll.com/comics/prince/andthesea.html $>$ (consultada el 8 de septiembre de 2020).

${ }^{20}$ Klintberg (2010), por ejemplo, dedica una sección de su catálogo de leyendas suecas (concretamente F51-60) a Erotic relations between water spirit and man.

${ }^{21}$ Última pàgina disponible en <http://emcarroll.com/comics/prince/12.html > (consultada el 8 de septiembre de 2020).

${ }^{22}$ Disponible en $<$ http://emcarroll.com/comics/snakeleaves/> (consultada el 8 de septiembre de 2020).

${ }^{23}$ Disponible en $<$ http://emcarroll.com/comics/faceallred/01.html $>$ (consultada el 8 de septiembre de 2020).
} 
señalado Schedeen, todos los cuentos de Through the Woods se acercan a la «manera de ser» de los cuentos:

All of these horror stories are period pieces. All of them have a distinctly fairy taleesque approach. All of them deal with the fear of unknown and unseen monsters lurking in the wilderness. So though this may be a collection of individual tales, Through the Woods has a very cohesive feel (Schedeen, 2015).

En este cuento, que es también un excelente ejemplo del uso del formato por parte de la autora (como se ha visto en la introducción), se explica el fratricidio del protagonista, que mata a su hermano cuando ambos salen a cazar a la bestia que aterroriza a todo el pueblo. La extraña reaparición del (supuesto) hermano levanta, como es lógico, las sospechas del protagonista, que deberá bajar a las profundidades del bosque (y con él, el lector) para descubrir la verdad. Como explica la propia autora, la inspiración de esta historia proviene de los cuentos, aunque no se trata de la versión de ningún relato concreto, sino más bien de la expresión de su espíritu, en una excelente aplicación práctica del concepto folkloresque:

At the same time, I'd written a fairy tale sort of story that dealt with two brothers hunting a beast in the woods, which resulted in one brother murdering the other for his own gain. And that, in turn, had been inspired by folktales where a character's act of betrayal catches up to him in the end (typically in some gruesome way) (Carroll entrevistada por Collins, 2011).

Sobre el final trágico de la historia, Carroll explica que «In most fairy tales where someone has caused some wrongdoing though, they pay for it by the story's end, which is (arguably, I suppose) what happens in "His Face"» (Carroll entrevistada por Collins, 2011). Se trata de la llamada «justicia inmanente» que podemos encontrar en determinados relatos folclóricos, como por ejemplo, las leyendas urbanas, en las que se transmite un mensaje moral que se presenta de manera indirecta señalando qué comportamiento debe seguirse. Según esta justicia inmanente, las personas que actúan mal son castigadas por las consecuencias de sus propios actos (Campion-Vincent \& Renard, 2002: 331-335).

\section{Conclusiones}

La obra de Emily Carroll presenta un universo propio cuya expresión se adecua, formalmente, el medio utilizado, ya sea este el digital (webcómic) o el papel (libro). En aquellos casos en que la misma obra aparece en los dos formatos, no se trata de una simple traslación sino de una adaptación a las características de cada medio. Del mismo modo, es posible establecer vínculos entre sus obras, a pesar de la aparente independencia de cada creación. No se trata, estrictamente hablando, de un único universo en el que conviven los diferentes personajes creados por Carroll, pero sí que se pueden establecer relaciones entre historias que comparten personajes o recursos utilizados.

La influencia del folclore en su obra, reconocida por la propia autora, puede tener dos tratamientos distintos. En primer lugar, las versiones folclóricas que recogen y adaptan (de maneras diferentes y en grados diversos) personajes o historias procedentes de la tradición oral (cuentos). Pertenecen a este primer grupo el webcómic The Hare's Bride que recuenta de manera explícita (autorizada) el cuento recogido por los hermanos 
Grimm («Häsichenbraut», Kinder- und Hausmärchen 66) y tres cuentos incluidos en Through the Woods. En dos de ellos, «Our Neighbor's House» (La casa del vecino) y «In Conclusion» (En resumen) se adapta, primero y recuenta, en segundo lugar, la historia de Caperucita Roja (Little Red Riding Hood, ATU 333), presentada, de esta manera, bajo un nuevo prisma por parte de la autora que acentúa, en este sentido, el componente terrorífico del relato por encima de su ejemplaridad. «A Lady's Hands are Cold» (La dama de las manos frías), por otro lado, es una adaptación mucho más libre del cuento Maiden-Killer (Bluebeard) (ATU 312) ya que la relación establecida es puramente una alusión que demanda más implicación por parte del lector para poder realizar este reconocimiento (siempre que, como es obvio, sea conocedor del relato tradicional). El uso de la tipografía y, sobre todo, del color (azul) por parte de la autora resultan, en este sentido, claves para poder realizar esta identificación. El libro Baba Yaga's Assistant, en el cual Carroll se ocupa únicamente de la parte gráfica, ejemplifica un uso combinado de la relación entre la fuente original y su recreación, ya que se trata de una historia que adapta (y no recuenta) de manera explícita (autorizada) el conocido personaje del folclore eslavo. En esta ocasión, pero, con un tono mucho más amable (y nada terrorífico) al tratarse de una publicación dirigida al público infantil y juvenil (y no adulto, como en la obra propia de Carroll).

El segundo tratamiento que se puede encontrar en la obra de Carroll en relación con el folclore son las llamadas versiones «folclorescas», no ligadas a ningún relato concreto, pero que presentan elementos que dotan de una apariencia folclórica (popular) a su obra aunque no tengan ese origen. Pertenecen a este segundo grupo tres webcómics (Anu-Anulan \& Yir's Daughter, The Prince \& The Sea y The Three Snake Leaves) que no adaptan ningún relato tradicional pero que utilizan los recursos propios de los mismos (a nivel argumental o gráfico) para adoptar una apariencia de cuento folclórico que parece proceder de alguna tradición cultural existente, cuando, en realidad, procede de la imaginación de su autora (y de sus influencias recibidas, cualesquiera sea el origen de las mismas). El cuento «His Face All Red» (Y la cara toda roja), publicado inicialmente en webcómic e incluido posteriormente en el libro Through the Woods, forma parte de este tipo de relatos construidos a la manera del folclore (y percibidos de este mismo modo por parte del lector) y es, a su vez, un magnífico ejemplo de como Carroll adapta la representación gráfica de sus historias según el medio utilizado (webcómic o papel). En este sentido, la aplicación del concepto de folcloresco a la obra de la autora canadiense resulta súmamente útil ya que permite relacionarla con elementos propios de la tradición que Carroll utiliza de manera libre y a conveniencia en cada historia.

La obra de Emily Carroll no se explica, pues, sin la influencia del folclore, pasado, eso sí, por su tamiz personal, y que deriva en una producción artística reconocible (gráficamente) pero también estilísticamente, con una fijación por la vertiente más terrorífica de los relatos y que ya es, a dia de hoy, una marca que permite identificar a primera vista su obra. Las escenas impactantes y los finales abruptos, que consiguen atrapar y sorprender al lector, ponen de manifiesto este universo particular que cuenta, cada día, con más seguidores que esperan, con deleite, de qué manera Carroll los sorprenderá (y aterrorizará) con su próxima creación. De la misma manera, cómo se ha visto, la influencia de los cuentos resulta evidente en muchas de sus obras, no solo de manera directa (como sucede con las versiones folclóricas que derivan directamente de un relato tradicional) sino también en una parte de su producción artística que reproduce argumentos, esquemas y elementos (narrativos, pero también gráficos) que recuerdan el folclore, pero que son fruto de su propia imaginación, las llamadas versiones «folclorescas». 


\section{BibliografíA}

Aldred, B. Grantham (2016): «Graphic novel», en Folktales and fairy tales: Traditions and texts from around the world, Anne E. Duggan \& Donald Haase con Helen J. Callow (eds.), $2^{\text {a }}$ ed., Santa Barbara, Greenwood, pp. 422-424.

Anderson, Laurie Halse \& CArroll, Emily (2018): Speak. The graphic novel, New York, Farrar Straus Giroux. (edición española: Cuéntalo, Barcelona, Ediciones La Cúpula, 2018)

Bender, Laureta \& Lourie, Reginald S. (1941): "The effect of comic books on the ideology of children», American Journal of Orthopsychiatry, 11 (3), pp. 540-550. DOI: https://doi.org/10.1111/j.1939-0025.1941.tb05840.x

CAmpion-Vincent, Véronique \& Renard, Jean-Bruno (2002): De source sûre. Nouvelles rumeurs d'aujourd'hui, Paris, Éditions Payot.

Carroll, Emily (2014): Through the Woods, London, Faber \& Faber. (edición española: Cruzando el bosque, Barcelona, Sapisti, 2015)

Carroll, Emily (2019): When I arrived at the castle, China, Koyama Press. (edición española: La noche que llegué al castillo, Barcelona, Sapristi, 2020)

Collins, Sean T. (2011): «Emily Carroll!» [Entrevista], The Comics Journal, accesible en $<$ http://www.tcj.com/emily-carroll/> (consultada el 8 de septiembre de 2020)

Doкоu, Christina (2017): «Un(th)inkable tales: Unimaginable folklore horror in Emily Carroll's Through the Woods», Journal of Graphic Novels and Comics, 8/6, pp. 572-587. DOI: https://doi.org/10.1080/21504857.2017.1383282

Foster, Michael Dylan (2016): «Introduction: The challenge of the folkloresque», en The folkloresque. Reframing folklore in a popular culture world, Michael Dylan Foster \& Jeffrey A. Tolbert (eds.), Colorado, Utah State University Press, pp. 3-33. DOI: https://doi.org/10.7330/9781607324188.c000

Gall, Elisa (2015): «Baba Yaga's Assistant», The Horn Book Magazine, septiembre/ octubre, pp. 109-110.

GRIMM, Brothers (2007): The complete fairy tales, translated, introduced and annotated by Jack Zipes, London, Vintage Books.

Horrocks, Sarah (2014): «Beautiful horror: Emily Carroll's Through the Woods is a comic masterwork», Comics Alliance, accesible en $<$ https://comicsalliance.com/emilycarroll-through-the-woods-review/> (consultada el 8 de septiembre de 2020)

KLintBerG, Bengt Af (2010): The types of the Swedish folk legend, Helsinki, Suomalainen Tiedeakatemia.

MAZur, Dan \& DANNER, Alexander (2017): «The international graphic novel», en The Cambridge companion to the graphic novel, Stephen E. Tabachnick (ed.), Cambridge, Cambridge University Press, pp. 58-79. DOI: https://doi. org/10.1017/9781316258316.006

Mccoola, Marika \& CArroll, Emily (2015): Baba Yaga's assistant, Shenzen, Candlewick Press.

Peretti, Daniel (2016): «Comics as Folklore», en The folkloresque. Reframing folklore in a popular culture world, Michael Dylan Foster \& Jeffrey A. Tolbert (eds.), Colorado, Utah State University Press, pp. 104-120. DOI: https://doi. org/10.7330/9781607324188.c004

SchedeEn, Jesse (2015): «Graphic novel review: Through the Woods», IGN, accesible en <https:/www.ign.com/articles/2015/01/16/graphic-novel-review-through-thewoods $>$ (consultada el 8 de septiembre de 2020) 
SiLva, Keith (2014): «Review: Through the Woods delivers timeless horror», Comics Bulletin, accesible en <http://comicsbulletin.com/woods/> (consultada el 8 de septiembre de 2020)

Sмith, Kevin Paul (2007): The postmodern fairytale: Folkloric intertext in contemporary fiction, Basingstoke, Palgrave Macmillan. DOI: https://doi. org/10.1057/9780230591707

UTHER, Hans-Jörg (2004) [ATU]: The types of international folktales. A classification and bibliography, Helsinki, Suomalainen Tiedeakatemia.

WeJebe, Alyssa (2019): «A lost comic?: Remembering Emily Carroll's 'Anu-Anulan and Yir's Daughter'», Goomba Stomp, accesible en $<$ https://goombastomp.com/a-lostcomic-remembering-emily-carrolls-anu-anulan-and-yirs-daughter/> (consultada el 8 de septiembre de 2020)

Whatman, Emma (2018): «Comics and graphic novels. Fairy-Tale Graphic Narrative», en The Routledge companion to media and fairy-tale cultures, Greenhill et al. (eds.), New York \& London, Routledge, pp. 474-482. DOI: https://doi.org/10. 4324/9781315670997-52

Fecha de recepción: 9 de septiembre de 2020

Fecha de aceptación: 5 de diciembre de 2020

$$
\text { i }
$$

